\title{
Repurposing metformin for the prevention of cancer and cancer recurrence
}

\author{
Brandy M. Heckman-Stoddard ${ }^{1}$ - Andrea DeCensi ${ }^{2,3}$ - Vikrant V. Sahasrabuddhe ${ }^{1}$. \\ Leslie G. Ford ${ }^{1}$
}

Received: 2 June 2017 / Accepted: 8 June 2017 / Published online: 3 August 2017

(C) Springer-Verlag (outside the USA) 2017

\begin{abstract}
Multiple epidemiological studies have documented an association between metformin, used for treatment of type 2 diabetes, and reduced cancer incidence and mortality. Cell line models may not accurately reflect the effects of metformin in the clinical setting. Moreover, findings from animal model studies have been inconsistent, whilst those from more recent epidemiological studies have tempered the overall effect size. The purpose of this review is to examine metformin's chemopreventive potential by outlining relevant mechanisms of action, the most recent epidemiologic evidence, and recently completed and ongoing clinical trials. Although repurposing drugs with excellent safety profiles is an appealing strategy for cancer prevention and treatment in the adjuvant setting, there is no substitute for well-executed, large randomised clinical trials to define efficacy and determine the populations that are most likely to benefit from an intervention. Thus, enthusiasm remains for understanding the role of metformin in cancer through ongoing clinical research.
\end{abstract}

Keywords Cancer - Cancer prevention - Cancer recurrence · Chemoprevention · Metformin · Review

Brandy M. Heckman-Stoddard

heckmanbm@mail.nih.gov

1 Division of Cancer Prevention, National Cancer Institute, 9609 Medical Center Drive, Bethesda, MD 20892, USA

2 Division of Medical Oncology, Ente Ospedaliero Ospedali Galliera, Genoa, Italy

3 Wolfson Institute of Preventive Medicine, Queen Mary University of London, London, UK

\author{
Abbreviations \\ ACF Aberrant crypt foci \\ AMPK AMP-activated protein kinase \\ CAV1 Caveolin-1 \\ HNSCC Head and neck squamous cell carcinoma \\ MCT4 Monocarboxylate transporter 4 \\ mTOR Mammalian target of the rapamycin \\ NSAID Non-steroidal anti-inflammatory drug \\ OCT Organic cation transporter \\ pS6K Phospho-serine 6 kinase \\ SRR Summary RR
}

\section{Introduction}

Metformin, a biguanide, is the first-line treatment for type 2 diabetes mellitus as an oral glucose-lowering agent. It decreases hepatic gluconeogenesis and improves insulin sensitivity by increasing peripheral glucose uptake and use, lowering both basal and postprandial plasma glucose [1]. It was first approved in the UK in 1958, followed by Canada in 1972, and then the USA in 1994 [2]. The most common side effects that occur in $>5 \%$ of patients include diarrhoea, nausea/ vomiting, flatulence, indigestion, abdominal discomfort, lack of energy and headache [1]. Lactic acidosis is a rare but potentially fatal side effect that can be avoided by checking and monitoring renal function. This tolerable and safe profile, in addition to its low cost as a generic drug, has made metformin an excellent candidate for repurposing. The ease of access to metformin has led to a multitude of preclinical and clinical investigations, as a single agent and in combination with other agents, for both cancer prevention and treatment. According to Web of Science (http://wok.mimas.ac.uk, accessed 15 March 2017), publications on 'metformin' AND 'cancer' have increased from $<40$ in 2008 to almost 500 per year in 
2015 and 2016, with more than 12,000 citations in 2016. In this review, we provide an update of the recent progress in this field and provide insights into the clinical development of metformin as both a preventive and adjuvant intervention for cancer.

\section{Molecular mechanisms}

The potential molecular mechanisms of metformin in cancer prevention and treatment have been extensively reviewed. Metformin is thought to have two potential routes of action that contribute to its anti-neoplastic activity: (1) an indirect route related to its insulin-lowering activity, which may slow tumour proliferation in individuals with hyperinsulinaemia; and (2) direct action in target tissues against respiratory complex I of the electron transport chain in mitochondria of preneoplastic and neoplastic cells, reducing energy consumption in the cell [3]. Both routes of action involve the activation of AMP-activated protein kinase (AMPK) by metformin, which inhibits the mammalian target of the rapamycin (mTOR) pathway, reducing cell proliferation, and inducing apoptosis and cell-cycle arrest $[4,5]$. Depending on the target organ, the mechanism of metformin action may be direct, indirect or a combination of the two.

Indirect action of metformin involves the insulin/IGF-1 pathway, which is activated in a setting of nutrient availability [6]. By activating both the phosphatidylinositol-4,5bisphosphate 3-kinase (PI3K)/Akt/mTOR and the Ras/Raf/ mitogen-activated protein kinase (MAPK) pathways, insulin/ IGF-1 activity contributes to increased cell growth and proliferation. Metformin decreases hepatic glucose output, increasing muscle uptake of glucose and reducing plasma insulin levels, which in turn may reduce proliferation of both preneoplastic and neoplastic cells. Metformin may also affect cancer cells indirectly through modulation of the immune response [7].

Metformin is hydrophilic $(\log D$ [where $D$ is distributioncoefficient] of 26.13 at $\mathrm{pH}$ 6.0) and charged at all physiological $\mathrm{pH}$ values ( $\mathrm{p} K_{\mathrm{a}}$ [the logarithmic constant of the acid dissociation constant $\left.\left(K_{\mathrm{a}}\right)\right]$ is 12.4 ), requiring transporters to cross the cell membrane for direct action [8]. These include the organic cation transporters (OCT) 1-3, plasma monoamine transporter (PMAT) and the multidrug and toxin extrusion protein (MATE) 1 and 2 [1,9]; OCT1 is critical for uptake into hepatocytes [10]. The expression of metformin transporters is variable across tumour cell lines and has been shown to be downregulated in breast tumour tissues in comparison with adjacent nonmalignant tissues [11]. In human cancer cell lines, which are known to express OCT1, metformin has been shown to decrease cell proliferation through inhibition of complex I [12]. However, often the transporter expression is not examined in these types of mechanistic, as well as clinical, studies. Furthermore, experiments involving a broad range of cancer cell lines grown in a low-glucose environment suggested that defects in glucose utilisation and mitochondrial function may predict sensitivity to metformin [13]. In women undergoing surgery for ovarian cancer who were taking metformin vs matched women who were not taking metformin, using metabolomics investigators showed metformin to be present in the ovarian tissue along with altered mitochondria metabolism, suggesting a direct effect of metformin on tumours, at clinical doses [14].

In addition to the role of metformin in cellular proliferation and apoptosis, metformin has been shown to inhibit many other cancer pathways. This includes blocking invasion of tumour cells by inhibiting matrix metalloproteinase- 9 activation [15], activating growth suppressors through phosphorylation of retinoblastoma protein $\left(\mathrm{pRb}\right.$ ) resulting in $\mathrm{G}_{0} / \mathrm{G}_{1}$ arrest in prostate cancer cells [16] and inhibiting cancer stem-cell activity [17].

\section{Epidemiological evidence}

A substantial body of observational studies, reviewed in numerous systematic reviews, have examined the effect of metformin on overall cancer incidence and mortality, as well as individual cancer sites. Multiple meta-analyses of case-control and cohort studies have now reported a decrease in overall cancer incidence of approximately 10 to $40 \%$ with metformin use, along with a decrease in mortality by a similar range [18-28] (Table 1, modified from [29]). In contrast, metaanalyses of RCTs have shown a non-significant change in cancer incidence [22-24, 28]; however, the randomised trials included were conducted to treat diabetes or reduce cardiovascular events and had baseline median ages ranging from 47 to 60 and short follow-up time, making them underpowered to detect an effect on cancer incidence. We also performed a meta-analysis, paying particular attention to biases and confounders following a review by Suissa and Azoulay underscoring the prevalence of time-related biases in observational studies and their potential to inflate estimates of the protective effect of metformin [30]. Similar to prior studies, we found the overall reduction of cancer incidence with metformin use was 30 to $40 \%$; however, excluding time-biased studies, the reduction in overall cancer incidence was only $10 \%$, albeit still statistically significant [24].

Metformin has been associated with a decreased risk of breast, colon, liver, pancreas, prostate, endometrium and lung cancer across meta-analyses. Besides time bias, individual organ-specific confounders are also important. For lung cancer, overall and time-unbiased analyses point to a protective effect of metformin with a summary RR (SRR) of 0.82 (95\% CI $0.67,0.99$ ), whereas adjustment for smoking, the leading cause of lung cancer, resulted in loss of significance with an 
Table 1 Meta-analyses examining the effect of metformin use on cancer incidence and mortality

\begin{tabular}{lll}
\hline Study authors (date) & $\begin{array}{l}\text { Association of } \\
\text { metformin use with } \\
\text { overall cancer } \\
\text { incidence, } \\
\text { SRR }(95 \% \text { CI })\end{array}$ & $\begin{array}{l}\text { Association of } \\
\text { metformin use } \\
\text { with overall cancer } \\
\text { mortality, SRR } \\
(95 \% \text { CI })\end{array}$ \\
\hline DeCensi et al (2010) [18] & $0.68(0.52,0.88)$ & $0.70(0.51,0.96)$ \\
Noto et al (2012) [20] & $0.67(0.53,0.85)$ & $0.66(0.49,0.88)$ \\
Soranna et al (2012) [21] & $0.61(0.54,0.70)$ & ND \\
Stevens et al (2012) [28] & $1.02(0.82,1.26)^{\mathrm{a}}$ & $\mathrm{ND}$ \\
Thakkar et al (2013) [22] & $1.01(0.81,1.26)^{\mathrm{a}}$ & $\mathrm{ND}$ \\
& $0.70(0.67,0.73)^{\mathrm{b}}$ & \\
& $0.90(0.84,0.98)^{\mathrm{c}}$ & \\
Franciosi et al (2013) [19] & $0.98(0.81,1.19)^{\mathrm{a}}$ & $0.65(0.53,0.80)$ \\
& $0.73(0.61,0.88)$ & \\
Zhang et al (2013) [25] & $0.73(0.64,0.83)$ & $0.82(0.76,0.89)$ \\
Lega et al (2014) [26] & ND & $0.74(0.62,0.88)$ \\
Zhang and Li (2014) [27] & ND & $0.70(0.55,0.88)$ \\
Gandini et al (2014) [24] & $0.69(0.52,0.90)$ & $0.66(0.54,0.81)$ \\
& $0.95(0.69,1.30)^{\mathrm{a}}$ & \\
& $0.90(0.89,0.91)^{\mathrm{d}}$ & \\
& $0.86(0.83,0.90)$ & $0.70(0.53,0.94)$ \\
Wu et al (2015) [23] & $1.05(0.94,1.18)^{\mathrm{a}}$ & $0.91(0.37,2.23)^{\mathrm{a}}$ \\
& $0.88(0.83,0.92)^{\mathrm{b}}$ & $0.66(0.49,0.89)^{\mathrm{b}}$ \\
$0.71(0.63,0.80)^{\mathrm{c}}$ & \\
\hline
\end{tabular}

Table modified from [26]

${ }^{\mathrm{a}} \mathrm{RCT}$

${ }^{\mathrm{b}}$ Cohort study

${ }^{\mathrm{c}}$ Case-control study

${ }^{\mathrm{d}}$ Adjusted for time bias

ND, no data

SRR of 0.95 (95\% CI 0.82, 1.11). A recent meta-analysis that focused on the role of metformin and colorectal adenoma, a precursor to colorectal cancer, showed a pooled OR of 0.76 (95\% CI 0.63, 0.92). However, they also noted publication bias against negative studies [31]. Metformin use has previously been shown to be associated with a reduced risk of colorectal cancer [32-34].

Compared with cancer incidence, the effect of metformin on cancer mortality is informed by fewer studies and, thus, site-specific analyses of the effects of metformin on overall mortality have not been performed in older meta-analyses [24]. However, more recent meta-analyses have focused on the impact of metformin on site-specific cancer mortality and overall survival, as reviewed in Table 2 [26, 27, 35-43]. These analyses have shown marked reductions in cancerspecific mortality for colon, lung and early-stage prostate cancer, and improvements in overall survival for breast, colon, gynaecological (endometrial and ovarian), liver, lung, prostate and pancreatic cancer.

Taken together, these results point out the inconsistent evidence from epidemiological studies that often do not completely capture or misclassify important information prior to analysis, such as the dose and duration of metformin therapy, diabetes history (duration and progression diabetes), changes in glucose-lowering medications, and the potential that participants may be taking multiple combined glucoselowering medications [44]. Therefore, while most studies, including our meta-analysis, suggest that metformin use is associated with a reduced risk of cancer and death from cancer, the effect size may be smaller than previously believed.

\section{Reported clinical trials}

To date, there are 17 clinical trials that have examined the effect of metformin on cancer related biomarkers and reported results (Table 3; adapted from [29]). The studies were conducted in various disease settings, spanning at-risk participants, individuals with preneoplastic lesions, individuals who were awaiting surgical excision, and those receiving metformin in an adjuvant setting.

A multicentre randomised trial of metformin in individuals with Barrett's oesophagus who were taking a proton pump inhibitor showed no significant change in phospho-serine 6 kinase (pS6K), a biomarker of insulin pathway activation, comparing baseline endoscopy biopsies with end of study biopsies [45]. Non-steroidal anti-inflammatory drug (NSAID) use was allowed in the study to aid patient accrual. Since NSAIDs are associated with decreased cancer risk, this may have affected the study's ability to observe an effect.

Two studies examined various doses of metformin given for 3 to 6 months to women after completion of chemotherapy and radiation for breast cancer $[41,42]$. The first study examined specifically selected women whose plasma levels of insulin were at least $45 \mathrm{pmol} / 1$ [46]. This group was selected based on a prior cohort study that showed women with these characteristics were at an increased risk of breast cancer [47]. In this study, metformin decreased circulating insulin levels by $22.4 \%(p=0.024)$ [46]. The second study enrolled women with elevated testosterone levels and compared two doses of metformin ( $1000 \mathrm{mg} /$ day vs $1500 \mathrm{mg} /$ day $)$; the higher dose significantly reduced serum testosterone levels and free androgen index compared with the lower dose [48]. These findings demonstrate that metformin could reduce serum markers associated with breast cancer risk.

Four trials have examined the short-term effects (1-4 weeks) of various doses of metformin on cell proliferation (as assessed by the expression of Ki-67) in tissues from women awaiting surgery for breast cancer (presurgical trials) [44-47]. One of these trials found a $3.4 \%$ reduction in $\mathrm{Ki}-67(p=0.027)$ in the metformin arm, comparing biopsies at diagnosis with biopsies at the time of surgery [49]. However, in the largest randomised, double-blind, placebo-controlled study of the effect of metformin on $\mathrm{Ki}-67$ expression in breast cancer, the change in $\mathrm{Ki}-67$ 
Table 2 Meta-analyses examining the effect of metformin use on cancer site-specific mortality

\begin{tabular}{llll} 
Cancer Site & Study authors (date) & $\begin{array}{l}\text { Association of metformin } \\
\text { use with cancer site-specific } \\
\text { mortality, HR }(95 \% \text { CI })\end{array}$ & $\begin{array}{l}\text { Association of metformin } \\
\text { use with overall mortality, } \\
\text { HR (95\% CI) }\end{array}$ \\
\hline Breast & Yang et al (2015) [35] & ND & $0.70(0.51,0.96)$ \\
Colon & Lega et al (2014) [26] & $0.65(0.56,0.76)$ & ND \\
& Zhang and Li (2014) [27] & ND & $0.70(0.59,0.84)$ \\
Endometrial & Coyle et al (2016) [39] & $0.58(0.39,0.86)$ & $0.69(0.58,0.83)$ \\
Liver & Perez-Lopez et al (2017) [36] & ND & $0.64(0.45,0.89)$ \\
Lung & Ma et al (2016) [37] & ND & $0.59(0.42,0.83)$ \\
& Wan et al (2016) [38] & $0.65(0.52,0.83)$ & $0.78(0.64,0.93)$ \\
Ovarian & Tian et al (2016) [42] & ND & $0.90(0.84,0.96)$ \\
Prostate & Zhang and Li (2014) [27] & ND & $0.44(0.30,0.64)$ \\
& Raval et al (2015) [43] & $0.76(0.43,1.33)$ & $0.86(0.67,1.10)$ \\
& Coyle et al (2016) ${ }^{\mathrm{a}}[39]$ & $0.58(0.37,0.93)$ & $0.82(0.73,0.93)$ \\
Pancreas & Stopsack et al (2016) [40] & $0.76(0.44,1.31)$ & $0.88(0.86,0.90)$ \\
\hline
\end{tabular}

${ }^{\text {a }}$ Study focused on localised, early-stage disease

$\mathrm{ND}$, no data between diagnostic biopsy and surgical specimen was not significant relative to placebo [50]. Although, women with higher insulin resistance (HOMA-IR >2.8) had a non-significant decrease in $\mathrm{Ki}-67$ expression of $10.5 \%$, while women with no/lower insulin resistance (HOMA-IR <2.8) had a nonsignificant increase in expression $(11.1 \%)$. The interaction between insulin resistance (as measured by the HOMA-IR) and metformin with regard to $\mathrm{Ki}-67$ expression was statistically significant $(p=0.045)$ [50]. A third, single-arm trial reported a decrease in Ki-67 levels, from $36.5 \%$ to $33.5 \%(p=0.016)$, after a median of 18 days of metformin treatment (diagnostic biopsy vs surgical specimen) [51], while a recently completed study in a majority Hispanic population with a historical control group matched for age, BMI and stage, showed no reduction in $\mathrm{Ki}-67$ in the metformin arm or in the untreated control group, when baseline biopsies were compared with surgical excisional specimens [52].

Multiple studies have examined the effect of metformin on colon cancer prevention. The number of aberrant crypt foci (ACF; a putative precursor of colon cancer) was examined in individuals with pre-existing ACF, randomising participants to very low dose metformin (250 $\mathrm{mg}$ /day) or no treatment for 1 month [53]. A significant decrease in ACF (mean \pm SD), from $8.78 \pm 6.45$ to $5.11 \pm 4.99(p=0.007)$, was observed in the metformin arm but not in the control group. A follow-up Phase III, multicentre, double-blind, placebo-controlled trial performed by the same group randomised participants with a history of colorectal adenoma to either metformin at $250 \mathrm{mg} /$ day $(n=151)$ or placebo $(n=143)$ for 1 year. Seventy-one participants in the metformin arm and 62 in the placebo group had an end-of-study colonoscopy and the RR of adenomas was 0.60 (95\% CI 0.39, 0.92) [54]. A multicentre single-arm study also examined a similar population with a history of adenoma in the previous 3 years, with $\mathrm{BMI} \geq 30 \mathrm{~kg} / \mathrm{m}^{2}$. Participants were given metformin at an escalating dose, from 500 to $2000 \mathrm{mg}$ /day for 12 weeks. No significant changes in the primary endpoint, pS6K levels, were observed (ClincalTrials.gov registration no. NCT01312467).

The role of metformin in endometrial cancer prevention is of particular interest because of the strong association between endometrial cancer and obesity, and the use of metformin for the treatment of polycystic ovary syndrome, a common condition that is associated with increased risk of endometrial cancer. Four studies have been reported in women with early-stage endometrial cancer and/or endometrial hyperplasia who were treated with metformin prior to hysterectomy [51-55]. Among women with Stage 1-2 endometrioid endometrial cancer and a BMI $\geq 30 \mathrm{~kg} / \mathrm{m}^{2}$, treatment with $850 \mathrm{mg} /$ day metformin resulted in an $11.75 \%$ reduction in Ki-67 ( $p=0.008)$ [55]. In addition, using serum metabolomics, this study showed a greater modulation in lipid metabolism among metformin responders vs non-responders, which the authors suggest support an indirect mechanism of metformin action on cell proliferation [55]. Another study in individuals with endometrioid endometrial cancer with dosage escalation of metformin from $750 \mathrm{mg} /$ day to $1500 \mathrm{mg}$ /day or $2250 \mathrm{mg}$ /day (based on the individual's tolerance of metformin), for 4 weeks prior to surgery showed a $44.2 \%$ decrease in Ki-67 with metformin use $(p<0.001)$, with no significant change in the untreated historical control group [56]. This study also examined the concentration of metformin in endometrial tissue, which was found to be $1.2-5.1 \mu \mathrm{mol} / \mathrm{kg}$ wet weight (approximately $20 \%$ of that in plasma), demonstrating that metformin was present in the endometrium [56]. 


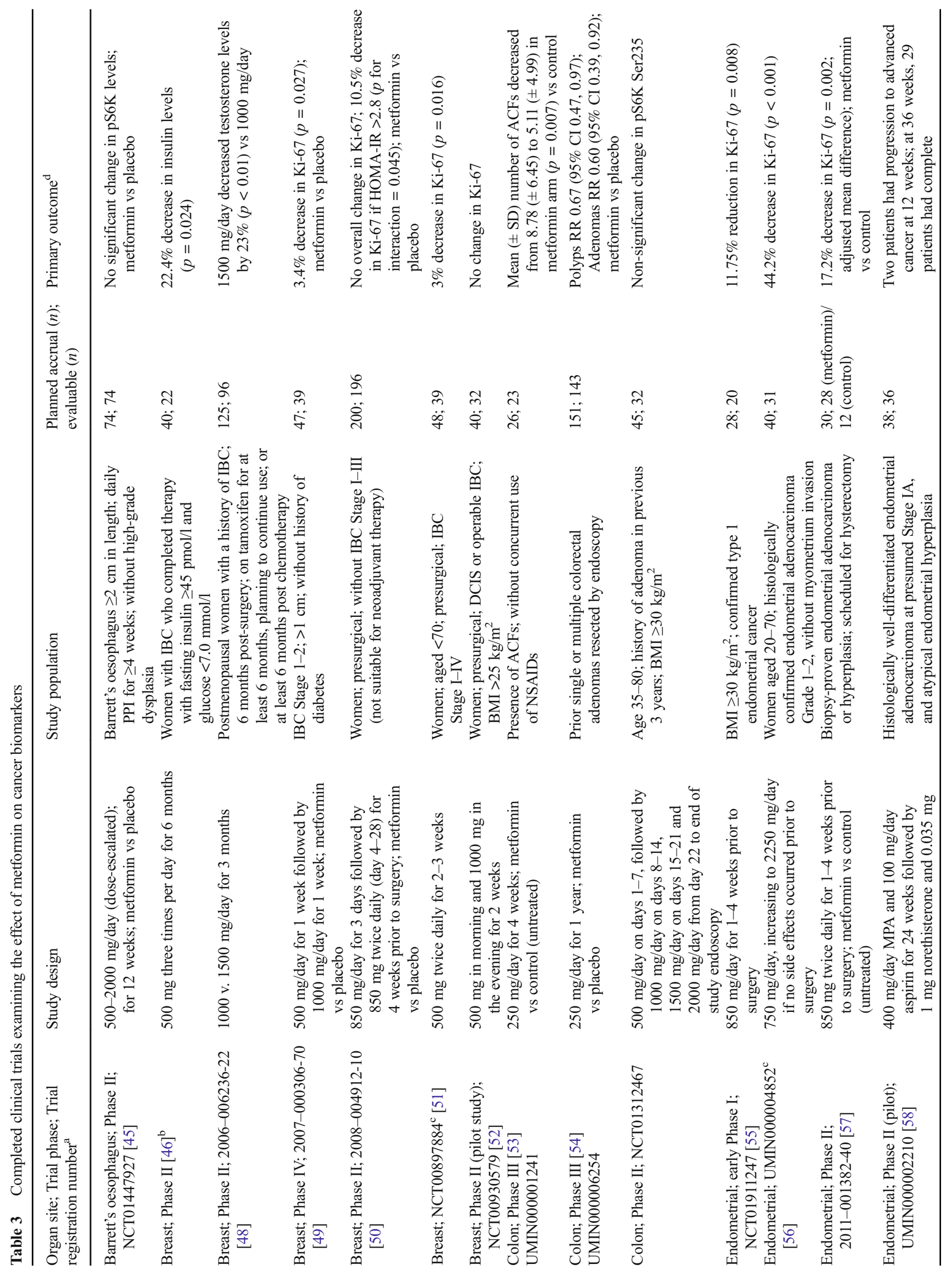




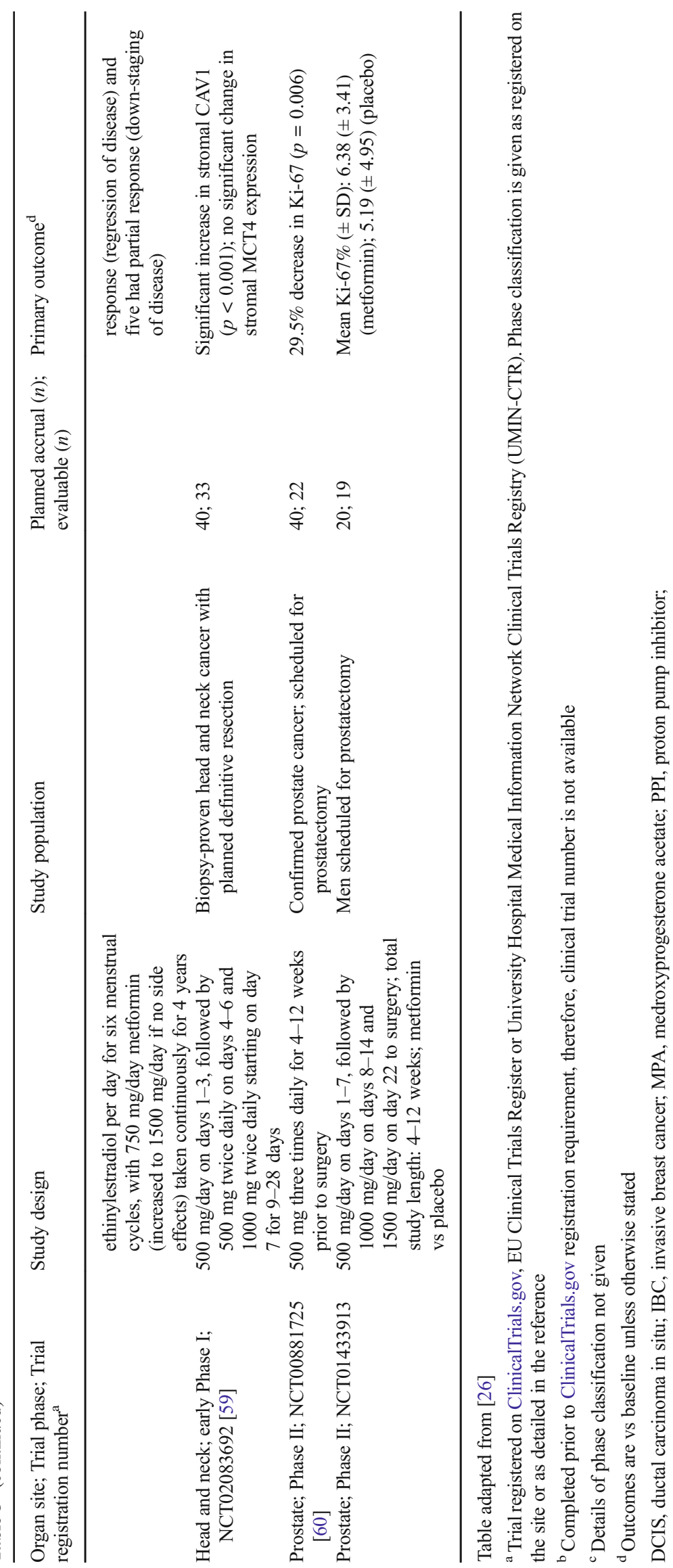


In a non-randomised study of women with biopsy-proven endometrial hyperplasia or endometrioid endometrial cancer, 28 women were treated with $850 \mathrm{mg}$ of metformin twice daily, while 12 women received no drug. A significant adjusted mean difference in $\mathrm{Ki}-67$ of $-17.2 \%(p=0.002)$ was observed in the metformin group vs control group [57]. Another study examined fertility preservation in women with endometrial hyperplasia or Grade 1 endometrial cancer using dose-escalated metformin therapy, from $750 \mathrm{mg} /$ day to $1500 \mathrm{mg} /$ day, in combination with $400 \mathrm{mg}$ /day medroxyprogesterone acetate and $100 \mathrm{mg}$ /day aspirin for 24 weeks, followed by a low-dose monophasic agent (1 mg norethisterone and $0.035 \mathrm{mg}$ ethinylestradiol per day) for six menstrual cycles [58]. Two participants showed progression to advanced cancer at 12 weeks, while 29 achieved a complete response (regression of disease) and five had a partial response (down-staging of disease) at 36 weeks, with 16 of these individuals going on to conceive [58].

A single study has examined the role of metformin in head and neck squamous cell carcinoma (HNSCC) prior to surgery, with change in stromal caveolin-1 (CAV1) and monocarboxylate transporter 4 (MCT4) as the primary endpoint, with the rationale that metformin targets cells with altered glycolysis, which has been shown in cancer-associated fibroblasts particularly in HNSCC [59]. Low CAV1 and increased MCT4 levels are associated with aggressive disease in HNSCC. The study showed a significant increase in CAV1 but no change in MCT4 in 33 evaluable participants between baseline biopsy and surgical specimen [59].

Finally, two studies have evaluated metformin in men with prostate cancer treated with metformin $(500 \mathrm{mg}$ three times daily) for 4-12 weeks prior to surgery [56, 57]; a single-arm study examined change in $\mathrm{Ki}-67$, comparing diagnostic biopsy with tissue from prostatectomy, showing an absolute decrease in the Ki67 proliferation index (percentage of nuclei showing nuclear immunoreactivity of any intensity) of $1.44 \%$, which amounted to a statistically significant relative decrease from baseline of $29.5 \%$ $(p=0.006)$ [60]. The second randomised study demonstrated no difference in cell proliferation in prostatectomy samples between eight individuals in the metformin group (mean Ki-67, 6.38\%) at surgery compared with nine individuals in the placebo group at surgery (mean Ki-67, 5.19\%) (ClinicalTrials.gov registration no. NCT01433913). Baseline Ki-67 was not evaluated in this study. This study also evaluated metformin concentration in the prostate tissue, but the data are not yet published.

\section{Ongoing clinical trials}

An analysis of clinical trials registered on ClinicalTrials.gov (https://clinicaltrials.gov/, accessed 15 March 2017) revealed an additional 23 trials examining the effect of metformin in participants at risk of cancer, 30 presurgical studies and 30 studies in the adjuvant setting. This is a significant increase from a similar analysis of ongoing studies conducted in 2011, in which there were three studies examining the effect of metformin in participants at risk for cancer, nine presurgical studies and seven studies in the adjuvant setting. The ongoing studies examine similar endpoints, including pharmacodynamic markers, such as tumour/tissue levels of pS6K, or other measures of AMPK signalling. Many of the studies examine surrogate endpoints for cancer, such as atypia or breast density for breast cancer, or change in prostate specific antigen (PSA) for prostate cancer. As has been reported in many studies, accrual to clinical trials is challenging with many pilot studies closing because of poor accrual.

Building on results from early phase studies (described above) and other preclinical and observational epidemiological data, several Phase III studies are currently (2017) ongoing. For example, a Phase III randomized, placebo-controlled study in men with biopsy-proven, low-risk localised prostate cancer, undergoing active surveillance, is examining time to progression of prostate cancer with metformin (ClinicalTrial. gov registration no. NCT01864096). Further, the National Cancer Institute of Canada (NCIC) MA.32 trial, a Phase III adjuvant breast cancer trial, has randomised 3649 women within 12 months of diagnosis who have completed chemotherapy and radiation to metformin $850 \mathrm{mg}$ twice a day ( $850 \mathrm{mg}$ /day during weeks 1-4) vs placebo for 5 years (ClinicalTrial.gov registration no. NCT01101438). The primary endpoint is invasive disease-free survival. This study is fully accrued with an estimated completion date of July 2020. There was also a Phase III study planned in 408 individuals with hepatitis $\mathrm{C}$ liver cirrhosis, examining treatment with $1000 \mathrm{mg}$ twice daily metformin vs placebo for 36 months, with the primary endpoint of rate of hepatocellular carcinoma occurrence and liver-related death or transplantation. This study was started in June 2015 but was terminated at the decision of the investigator in April 2016 after 11 participants were accrued (ClinicalTrial.gov registration no. NCT02319200). The Diabetes Prevention Program was a Phase III study that started in 1996 to examine the influence of metformin and intensive lifestyle intervention compared with placebo on progression from impaired glucose tolerance to diabetes. The follow-up to this study (the Diabetes Prevention Program Outcomes Study [ClinicalTrial.gov registration no. NCT00038727]) has continued to actively follow this cohort, with open-label use of metformin in the original metformin arm, to examine progression to diabetes as well as the development of longterm diabetes complications. The study group has also retrospectively collected medical records for cancer that have been reported over the course of the study and will continue to collect data on cancer outcomes as part of an extension to the original clinical trial [61]. This study is unique because of the extensive length of longitudinal follow-up, the use of glucose tolerance tests to robustly identify disease (diabetes) 
onset, and availability of excellent data on dosing, exposure and compliance to metformin.

\section{Conclusions}

There is a long history and much clinical experience with metformin that makes it a very attractive candidate for drug repurposing for cancer prevention. Data on the potential mechanisms of metformin action for cancer prevention and treatment, as well as additional longitudinal epidemiological data from new and previously examined cohorts, continues to accumulate. There is biological plausibility for a cancer preventive effect of metformin, given multiple ways that it can interfere with cancer promoting signalling pathways. However, both animal and epidemiological studies have shown somewhat mixed effects. The epidemiological literature has largely evaluated individuals with diabetes, and the cancer-preventive effect is of lesser magnitude than previously reported once appropriate adjustments have been made to account for various biases and confounders. It remains to be determined whether a similar protective effect can be demonstrated in individuals without diabetes or in individuals with impaired fasting glucose. Multiple clinical trials have reported promising results, but Phase III studies are ongoing and these will provide the best evidence. Cancer is not one disease and each cancer subtype may respond to metformin in a unique way. Additional studies may be needed as data becomes available to elucidate those that are most likely to benefit from metformin treatment for the prevention of cancer and its recurrence.

Acknowledgements This material should not be interpreted as representing the viewpoint of the US Department of Health and Human Services, the National Institutes of Health, or the National Cancer Institute.

Funding Work in AD's laboratory is supported by grants from the Italian Association for Cancer Research (AIRC) and from TRANSCANERANET (JTC 2013-056).

Duality of interest The authors declare that there is no duality of interest associated with this manuscript.

Contribution statement All authors participated in drafting the article and revising it critically for important intellectual content. All authors approved the version to be published.

\section{References}

1. Zhou M, Xia L, Wang J (2007) Metformin transport by a newly cloned proton-stimulated organic cation transporter (plasma membrane monoamine transporter) expressed in human intestine. Drug Metab Dispos 35:1956-1962
2. Bailey C (2017) Metformin: historical overview. Diabetologia. doi: 10.1007/s00125-017-4318-z

3. Pollak MN (2012) Investigating metformin for cancer prevention and treatment: the end of the beginning. Cancer Discov 2:778-790

4. Han G, Gong H, Wang Y, Guo S, Liu K (2015) AMPK/mTORmediated inhibition of survivin partly contributes to metformininduced apoptosis in human gastric cancer cell. Cancer Biol Ther 16:77-87

5. Zhao D, Long XD, Lu TF et al (2015) Metformin decreases IL-22 secretion to suppress tumor growth in an orthotopic mouse model of hepatocellular carcinoma. Int J Cancer 136:2556-2565

6. Gonzalez-Angulo AM, Meric-Bernstam F (2010) Metformin: a therapeutic opportunity in breast cancer. Clin Cancer Res 16: $1695-1700$

7. Cameron AR, Morrison VL, Levin D et al (2016) Antiinflammatory effects of metformin irrespective of diabetes status. Circ Res 119:652-665

8. Saitoh R, Sugano K, Takata N et al (2004) Correction of permeability with pore radius of tight junctions in Caco-2 monolayers improves the prediction of the dose fraction of hydrophilic drugs absorbed by humans. Pharm Res 21:749-755

9. Hilgendorf C, Ahlin G, Seithel A, Artursson P, Ungell AL, Karlsson J (2007) Expression of thirty-six drug transporter genes in human intestine, liver, kidney, and organotypic cell lines. Drug Metab Dispos 35:1333-1340

10. Chen L, Shu Y, Liang X et al (2014) OCT1 is a high-capacity thiamine transporter that regulates hepatic steatosis and is a target of metformin. Proc Natl Acad Sci U S A 111:9983-9988

11. Cai H, Zhang Y, Han TK, Everett RS, Thakker DR (2016) Cationselective transporters are critical to the AMPK-mediated antiproliferative effects of metformin in human breast cancer cells. Int J Cancer 138:2281-2292

12. Wheaton WW, Weinberg SE, Hamanaka RB et al (2014) Metformin inhibits mitochondrial complex I of cancer cells to reduce tumorigenesis. Elife 3:e02242

13. Birsoy K, Possemato R, Lorbeer FK et al (2014) Metabolic determinants of cancer cell sensitivity to glucose limitation and biguanides. Nature 508:108-112

14. Liu X, Romero IL, Litchfield LM, Lengyel E, Locasale JW (2016) Metformin targets central carbon metabolism and reveals mitochondrial requirements in human cancers. Cell Metab 24:728-739

15. Bao B, Wang Z, Ali S et al (2012) Metformin inhibits cell proliferation, migration and invasion by attenuating CSC function mediated by deregulating miRNAs in pancreatic cancer cells. Cancer Prev Res 5:355-364

16. Ben Sahra I, Laurent K, Loubat A et al (2008) The antidiabetic drug metformin exerts an antitumoral effect in vitro and in vivo through a decrease of cyclin D1 level. Oncogene 27:3576-3586

17. Hirsch HA, Iliopoulos D, Tsichlis PN, Struhl K (2009) Metformin selectively targets cancer stem cells, and acts together with chemotherapy to block tumor growth and prolong remission. Cancer Res 69:7507-7511

18. DeCensi A, Puntoni M, Goodwin P et al (2010) Metformin and cancer risk in diabetic patients: a systematic review and meta-analysis. Cancer Prev Res 3:1451-1461

19. Franciosi M, Lucisano G, Lapice E, Strippoli GF, Pellegrini F, Nicolucci A (2013) Metformin therapy and risk of cancer in patients with type 2 diabetes: systematic review. PLoS One 8:e71583

20. Noto H, Goto A, Tsujimoto T, Noda M (2012) Cancer risk in diabetic patients treated with metformin: a systematic review and metaanalysis. PLoS One 7:e33411

21. Soranna D, Scotti L, Zambon A et al (2012) Cancer risk associated with use of metformin and sulfonylurea in type 2 diabetes: a metaanalysis. Oncologist 17:813-822

22. Thakkar B, Aronis KN, Vamvini MT, Shields K, Mantzoros CS (2013) Metformin and sulfonylureas in relation to cancer risk in 
type II diabetes patients: a meta-analysis using primary data of published studies. Metabolism 62:922-934

23. Wu L, Zhu J, Prokop LJ, Hassan Murad M (2015) Pharmacologic therapy of diabetes and overall cancer risk and mortality: a metaanalysis of 265 studies. Sci Rep 5:10147

24. Gandini S, Puntoni M, Heckman-Stoddard BM et al (2014) Metformin and cancer risk and mortality: a systematic review and meta-analysis taking into account biases and confounders. Cancer Prev Res 7:867-885

25. Zhang P, Li H, Tan X, Chen L, Wang S (2013) Association of metformin use with cancer incidence and mortality: a meta-analysis. Cancer Epidemiol 37:207-218

26. Lega IC, Shah PS, Margel D, Beyene J, Rochon PA, Lipscombe LL (2014) The effect of metformin on mortality following cancer among patients with diabetes. Cancer Epidemiol Biomark Prev 23:1974-1984

27. Zhang ZJ, Li S (2014) The prognostic value of metformin for cancer patients with concurrent diabetes: a systematic review and metaanalysis. Diabetes Obes Metab 16:707-710

28. Stevens RJ, Ali R, Bankhead CR et al (2012) Cancer outcomes and all-cause mortality in adults allocated to metformin: systematic review and collaborative meta-analysis of randomised clinical trials. Diabetologia 55:2593-2603

29. Heckman-Stoddard BM, Gandini S, Puntoni M, Dunn BK, DeCensi A, Szabo E (2016) Repurposing old drugs to chemoprevention: the case of metformin. Semin Oncol 43:123-133

30. Suissa S, Azoulay L (2012) Metformin and the risk of cancer: time-related biases in observational studies. Diabetes Care 35:2665-2673

31. Jung YS, Park CH, Eun CS, Park DI, Han DS (2017) Metformin use and the risk of colorectal adenoma: a systematic review and meta-analysis. J Gastroenterol Hepatol 32:957-965

32. He XK, Su TT, Si JM, Sun LM (2016) Metformin is associated with slightly reduced risk of colorectal cancer and moderate survival benefits in diabetes mellitus: a meta-analysis. Medicine 95:e2749

33. Nie Z, Zhu H, Gu M (2016) Reduced colorectal cancer incidence in type 2 diabetic patients treated with metformin: a meta-analysis. Pharm Biol 54:2636-2642

34. Rokkas T, Portincasa P (2016) Colon neoplasia in patients with type 2 diabetes on metformin: a meta-analysis. Eur J Intern Med 33:60-66

35. Yang T, Yang Y, Liu S (2015) Association between metformin therapy and breast cancer incidence and mortality: evidence from a meta-analysis. J Breast Cancer 18:264-270

36. Perez-Lopez FR, Pasupuleti V, Gianuzzi X, Palma-Ardiles G, Hernandez-Fernandez W, Hernandez AV (2017) Systematic review and meta-analysis of the effect of metformin treatment on overall mortality rates in women with endometrial cancer and type 2 diabetes mellitus. Maturitas 101:6-11

37. Ma SJ, Zheng YX, Zhou PC, Xiao YN, Tan HZ (2016) Metformin use improves survival of diabetic liver cancer patients: systematic review and meta-analysis. Oncotarget 7:66202-66211

38. Wan G, Yu X, Chen P et al (2016) Metformin therapy associated with survival benefit in lung cancer patients with diabetes. Oncotarget 7:35437-35445

39. Coyle C, Cafferty FH, Vale C, Langley RE (2016) Metformin as an adjuvant treatment for cancer: a systematic review and meta-analysis. Ann Oncol 27:2184-2195

40. Stopsack KH, Ziehr DR, Rider JR, Giovannucci EL (2016) Metformin and prostate cancer mortality: a meta-analysis. Cancer Causes Control 27:105-113

41. Zhou PT, Li B, Liu FR et al (2017) Metformin is associated with survival benefit in pancreatic cancer patients with diabetes: a systematic review and meta-analysis. Oncotarget 8:25242-25250

42. Tian RH, Zhang YG, Wu Z, Liu X, Yang JW, Ji HL (2016) Effects of metformin on survival outcomes of lung cancer patients with type 2 diabetes mellitus: a meta-analysis. Clin Transl Oncol 18: 641-649

43. Raval AD, Thakker D, Vyas A, Salkini M, Madhavan S, Sambamoorthi U (2015) Impact of metformin on clinical outcomes among men with prostate cancer: a systematic review and metaanalysis. Prostate Cancer Prostatic Dis 18:110-121

44. Bodmer M, Meier C, Krahenbuhl S, Jick SS, Meier CR (2010) Long-term metformin use is associated with decreased risk of breast cancer. Diabetes Care 33:1304-1308

45. Chak A, Buttar NS, Foster NR et al (2015) Metformin does not reduce markers of cell proliferation in esophageal tissues of patients with Barrett's esophagus. Clin Gastroenterol Hepatol 13:665-672

46. Goodwin PJ, Pritchard KI, Ennis M, Clemons M, Graham M, Fantus IG (2008) Insulin-lowering effects of metformin in women with early breast cancer. Clin Breast Cancer 8:501-505

47. Goodwin PJ, Ennis M, Pritchard KI et al (2002) Fasting insulin and outcome in early-stage breast cancer: results of a prospective cohort study. J Clin Oncol 20:42-51

48. Campagnoli C, Pasanisi P, Abba C et al (2012) Effect of different doses of metformin on serum testosterone and insulin in nondiabetic women with breast cancer: a randomized study. Clin Breast Cancer 12:175-182

49. Hadad S, Iwamoto T, Jordan L et al (2011) Evidence for biological effects of metformin in operable breast cancer: a pre-operative, window-of-opportunity, randomized trial. Breast Cancer Res Treat 128:783-794

50. Bonanni B, Puntoni M, Cazzaniga M et al (2012) Dual effect of metformin on breast cancer proliferation in a randomized presurgical trial. J Clin Oncol 30:2593-2600

51. Niraula S, Dowling RO, Ennis M et al (2012) Metformin in early breast cancer: a prospective window of opportunity neoadjuvant study. Breast Cancer Res Treat 135:821-830

52. Kalinsky K, Crew KD, Refice S et al (2014) Presurgical trial of metformin in overweight and obese patients with newly diagnosed breast cancer. Cancer Investig 32:150-157

53. Hosono K, Endo H, Takahashi H et al (2010) Metformin suppresses colorectal aberrant crypt foci in a short-term clinical trial. Cancer Prev Res 3:1077-1083

54. Higurashi T, Hosono K, Takahashi H et al (2016) Metformin for chemoprevention of metachronous colorectal adenoma or polyps in post-polypectomy patients without diabetes: a multicentre doubleblind, placebo-controlled, randomised phase 3 trial. Lancet Oncol $17: 475-483$

55. Schuler KM, Rambally BS, DiFurio MJ et al (2015) Antiproliferative and metabolic effects of metformin in a preoperative window clinical trial for endometrial cancer. Cancer Med 4:161-173

56. Mitsuhashi A, Kiyokawa T, Sato Y, Shozu M (2014) Effects of metformin on endometrial cancer cell growth in vivo: a preoperative prospective trial. Cancer 120:2986-2995

57. Sivalingam VN, Kitson S, McVey R et al (2016) Measuring the biological effect of presurgical metformin treatment in endometrial cancer. Br J Cancer 114:281-289

58. Mitsuhashi A, Sato Y, Kiyokawa T, Koshizaka M, Hanaoka H, Shozu M (2016) Phase II study of medroxyprogesterone acetate plus metformin as a fertility-sparing treatment for atypical endometrial hyperplasia and endometrial cancer. Ann Oncol 27:262-266

59. Curry J, Johnson J, Tassone P et al (2017) Metformin effects on head and neck squamous carcinoma microenvironment: window of opportunity trial. Laryngoscope. doi:10.1002/lary.26489

60. Joshua AM, Zannella VE, Downes MR et al (2014) A pilot / 'window of opportunity/' neoadjuvant study of metformin in localised prostate cancer. Prostate Cancer Prostatic Dis 17:252-258

61. The Diabetes Prevention Program Research Group (1999) The diabetes prevention program. Design and methods for a clinical trial in the prevention of type 2 diabetes. Diabetes Care 22:623-634 